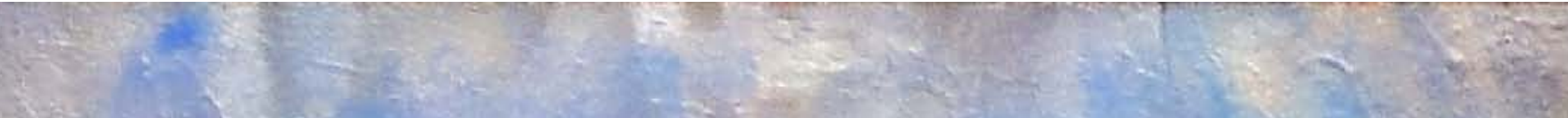

4.

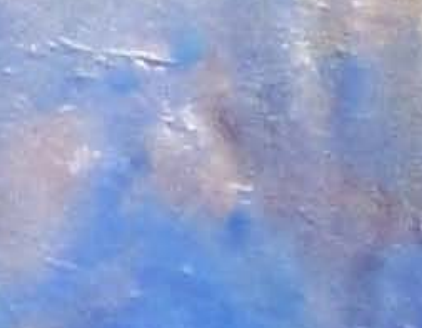

s.

$8+2=3$

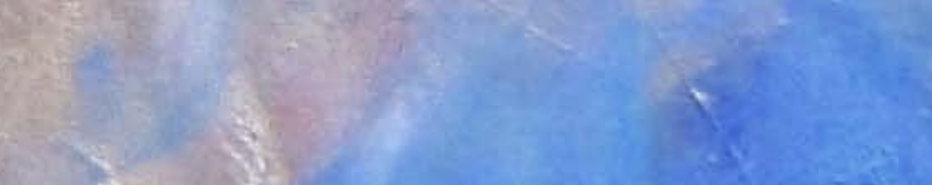

a 8

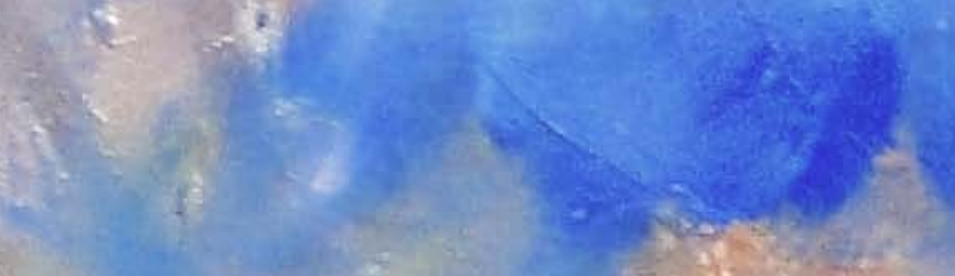
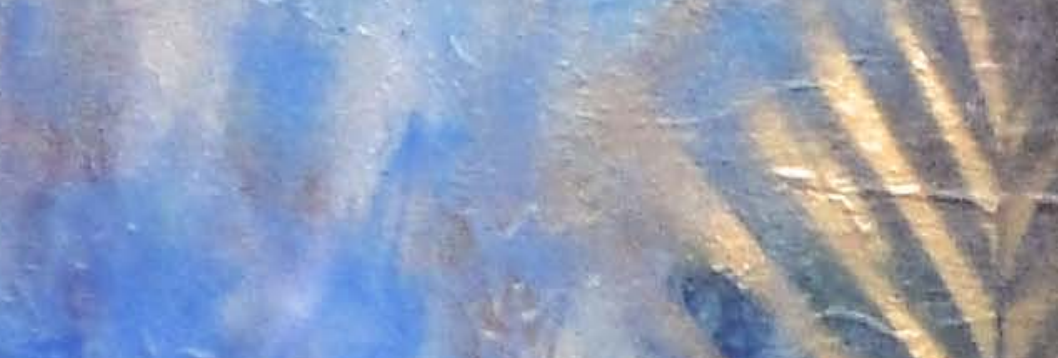
$3+x+3$

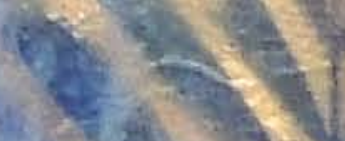

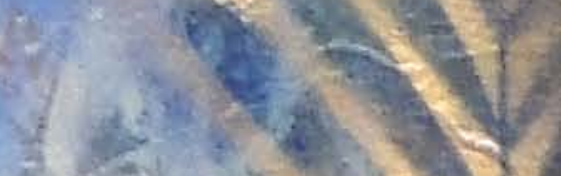

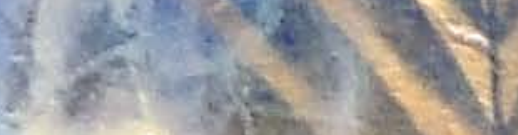

A. 


\section{Incidencia de embarazos de alto riesgo en el municipio de Langue, departamento de Valle}

JANINE GALLARDO*

RESUMEN. La Mancomunidad de Municipios Fronterizos "MAFRON" por convenio establecido con la Secretaría de Salud ha implementado en el municipio de Langue un proyecto piloto de descentralización de los servicios de salud, este permite en forma inmediata resolver problemas de abastecimiento de medicamentos, logística e insumos, así como recursos humanos en las diferentes unidades de salud, que integran la red de servicios en el municipio. A la vez este modelo permite a la comunidad participar de forma activa y social respecto al desempeño de los empleados y el uso de los recursos económicos utilizados en la unidad de salud, así como una mayor organización para contribuir en la gestión y administración de los servicios y en la conservación de la salud mediante la práctica de hábitos y estilos de vidas apropiados para los pobladores del municipio.

Palabras clave: descentralización, servicios de salud.

SUMMARY. The borderline Association of Municipalities Border "MAFRON" by agreement established with the Ministry of Health has implemented in the municipality of Langue a pilot project of health services decentralization, this allows to immediately solve problems of drug supply, logistics and supplies, as well as the lack of human resources in the health units which compose the services network in the municipality. Meanwhile this model allows the community to actively and socially participate regarding the performance of employees and the use of economic resources employed in the health unit, as well as a greater organization to assist in the management and administration of services and the preservation of health through the practice of habits and appropriate lifestyle for the residents of the municipality.

Keywords: decentralization, health services.

\section{Introducción}

Uno de los problemas de mayor preocupación para la Secretaría de Salud de Honduras es el área materno infantil; la cual presenta una problemática compleja en el tema de embarazos de alto riesgo.

El embarazo de alto riesgo es definido como aquel embarazo que tiene más probabilidades de presentar complicaciones tanto maternas como fetales, durante el transcurso de la gestación y el parto por lo que precisa de una mayor calidad de servicios de salud (Control médico y ecográfico estricto).

Esta investigación nace con el objetivo de conocer la problemática de los embarazos de alto riesgo y de esta manera identificar los grupos de edad que presentan el mayor porcentaje de embarazos de alto riesgo, ya que en el perfil epidemiológico existente del municipio de Langue, departamento de Valle la mortalidad materno infantil, está relacionada a factores de accesibilidad (Postergación geográfica, falta de educación, cultura, situación económica) y las necesidades reales de extensión y sostenibilidad de la cobertura de los servicios de salud.

El estudio se realizó mediante una investigación descriptiva entre las pacientes que asisten a cada unidad de salud del municipio de Langue y tiene como objetivo general presentar la problemática que se genera en torno al embarazo de alto riesgo en el municipio. 


\section{Metodología}

La investigación es descriptiva, hizo uso de fuentes de datos secundarias registrados por las unidades de salud del municipio de Langue a través de los expedientes clínicos y registros estadísticos de embarazos de alto riesgo entre los años 2007-2009. El universo lo constituyeron los casos de mujeres embarazadas que presentan características o rasgos de padecimiento de daños a la salud física y que asisten a control.

Una vez recolectada la información se vació en tablas diseñadas de acuerdo a lo establecido en los objetivos específicos, posteriormente se hizo el análisis de los datos dándole respuesta a los objetivos, comparando y analizando los resultados con la ayuda de gráficos y tablas.

\section{Contexto del estudio}

\subsection{Embarazos de alto riesgo}

Uno de los problemas que está afectando la salud de la población mundial en general y la hondureña en particular es la mortalidad materna. Cada año mueren alrededor de 108 mujeres por complicaciones relacionadas con el embarazo, el parto y el puerperio (USAID, 1998).

Los embarazos no planeados o no deseados elevan el riesgo para la salud: una de cada ocho muertes maternas se debe a problemas de salud relacionados con el aborto. Si no hubiera embarazos no deseados, se podrían evitar hasta 25\% de las muertes maternas. La planificación familiar también puede prevenir muertes infantiles: un niño o niña que nace con un espacio intergenésico menor de dos años, tiene el doble de probabilidades de morir que un niño o niña que nace con un intervalo de dos ó más años. De esta manera, se podrían evitar hasta 25\% de las muertes infantiles (Shane, 1997, tomada de Honduras Mejorar la Salud Reproductiva).

En Honduras, más de la mitad de los partos son atendidos en hospitales y centros de la Secretaría de Salud y se prevé que esta proporción aumente rápidamente en el futuro. Por otra parte, diversas investigaciones han mostrado que cuando hay métodos anticonceptivos en los hospitales y centros de salud, una proporción importante de mujeres elige un método antes de ser dadas de alta del hospital y que la prevalencia de uso de anticonceptivos seis meses después del parto es más elevada entre las mujeres que dan a luz en hospitales que cuentan con servicios de anticoncepción postparto/post aborto, que entre las mujeres que dan a luz en hospitales que no cuentan con estos servicios (Foreit et al, 1993 tomada de Honduras Mejorar la Salud Reproductiva).

En el municipio de Langue el perfil epidemiológico existente sobre la mortalidad materno-infantil, los factores de accesibilidad (postergación geográfica, cultural y económica) y las necesidades reales de extensión y sostenibilidad de cobertura de atención en salud continua y de calidad, justifica el esfuerzo institucional que realizan los entes gubernamentales para lograr un incremento de acceso a servicios de salud de la población. Ya que el embarazo en la adolescencia se asocia con las mayores tasas de morbilidad y mortalidad, tanto para la madre como para el niño o niña. La violencia es la segunda causa principal de muerte durante el embarazo para adolescentes y es mayor que en cualquier otro grupo.

Las adolescentes embarazadas tienen un riesgo mucho mayor de morir o sufrir complicaciones médicas graves como: placenta previa, hipertensión arterial inducida por el embarazo, parto prematuro, anemia grave y toxemia.

Los bebés nacidos de adolescentes tienen una probabilidad de 2 a 6 veces más de tener bajo peso al nacer que aquellos cuyas madres tienen 20 años o más. La prematuridad juega el mayor papel en el bajo peso al nacer, pero el retraso en el crecimiento intrauterino (crecimiento inadecuado del feto durante el embarazo) también es un factor.

Las madres adolescentes tienen más probabilidad de mostrar comportamientos malsanos, los cuales dejan al bebé en mayor riesgo de presentar crecimiento inadecuado, infección o dependencia de sustancias químicas. Igualmente, cuanto más joven sea la madre (por debajo de los 20 años), mayor será el riesgo para el bebé de morir durante el primer año de vida. El control prenatal oportuno y adecuado es de vital importancia para las adolescentes embarazadas. 
El embarazo en la adolescencia es de alto riesgo para la salud y vida de la mujer y del feto. Tiene implicaciones en el desarrollo del país ya que la fecundidad adolescente es más alta en países en desarrollo y en clases sociales menos favorecidas.

La proporción de jóvenes que han iniciado relaciones sexuales va en aumento. El embarazo en las adolescentes se ha convertido en una seria preocupación para varios sectores sociales:

- Sector de salud: por la mayor incidencia de resultados desfavorables. (Embarazos de alto riesgo).

- Sector social: por las consecuencias adversas que el hecho tiene sobre las adolescentes, familiares y comunidad.

- Sector educación: por el rendimiento académico, baja autoestima, desarrollo personal, etc.

La madre adolescente corre mayor riesgo de tener complicaciones durante el embarazo que las mujeres de más de 20 años. Entre la complicaciones más frecuentes están: parto prematuro, anemia y alta presión arterial. Estos riesgos son todavía mayores cuando tienen menos de 15 años. Estas madres tan jóvenes también tienen una doble probabilidad de morir a causa de complicaciones durante el embarazo en comparación con las madres entre los 20 y 24 años de edad.

Las siguientes constituyen las consecuencias que atraviesan la mayoría de las adolescentes embarazadas.

- Educativo: expulsión, pérdida del año escolar, discriminación y deserción escolar.

- Familiar: rechazo, recriminación, conflicto entre padres, aislamiento.

- Social: discriminación, abandono, aislamiento de los amigos, matrimonio forzado, madre-solterismo.

- Personal: pérdida de oportunidades educativas y laborales, madre-solterismo, matrimonio precoz, pérdida o aplazamiento de metas, baja autoestima, aislamiento, mayor riesgo de enfermedad y aborto, hijos no deseados y falta de afecto hacia ellos (Abandono), grandes responsabilidades a temprana edad. Las adolescentes embarazadas están en un mayor riesgo de contraer enfermedades de transmisión sexual estas enfermedades incluyen la clamidia (que puede causar esterilidad), la sífilis (que puede provocar ceguera, y la muerte de la madre o del bebé) y el VIH (el virus que causa el SIDA, que también puede ser fatal para ambos). En este contexto las unidades de salud del municipio han emprendido una serie de acciones encaminadas a la prevención de los embarazos a temprana edad (Ver anexo No. 1).

La Secretaría de Salud considera que el grupo de alto riesgo más vulnerable es el de riesgo obstétrico y perinatal relacionado con factores como: antecedentes de patología médica obstétrica general importante, antecedente de abuso sexual, enflaquecimiento (desnutrición), estatura de 1,50 m o menor, menarca a los 11 años o menos, actitud negativa o indiferente al inicio del embarazo, ser la mayor de los hermanos.

El embarazo de alto riesgo es aquel que se presenta en las mujeres embarazadas con características o cualidades de mayor probabilidad de sufrir daños en la salud. Hay algunos que son más frecuentes e importantes y que deben ser buscados y tratados por medio de la entrevista y a la vez dividirlos en riesgos psicosociales y biológicos, aunque estos siempre se asocian.

El enfoque de riesgo se caracteriza por ser:

- Anticipatorio: permitiendo aplicar medidas preventivas.

- Integral: abarcando los aspectos biológicos, psicológicos y sociales de la mujer.

El enfoque de riesgo en la adolescente es muy importante ya que los comportamientos ante la morbimortalidad predominante en la adolescencia comparten la toma de riesgo y hay que investigarlos sistemáticamente, por lo que requiere de un equipo interdisciplinario.

A continuación se presentan los dos tipos de factores de riesgo que puede padecer una mujer embarazada:

\section{Factores psicosociales de riesgo}

- Asicronía madurativa físico emocional

- Baja autoestima

- Bajo nivel de instrucción 
- Pérdida reciente de personas significativas

- Embarazo por violación o abuso intento de aborto

- Propósito de entregar el hijo en adopción

- Familia disfuncional o ausente, sin adulto referente

- Trabajo no calificado

- Pareja ambivalente o ausente

- Condición económica desfavorable

- Marginación de su grupo de pertenencia

- Difícil acceso a los centros de salud

- Escaso espacio social frente al problema, con autoridades escolares que la separan de la institución, empleadores que la despiden y dificultades para constituir una familia.

\section{Factores de riesgo biológico}

- Edad cronológica < 14 años y / o edad ginecológica $<$ 1 año

- Peso $<45 \mathrm{Kg}$ y / o talla $<1,45 \mathrm{~m}$

- Estado nutricional deficiente

- Aumento insuficiente de peso para su biotipo y estado pre-concepción

- Hábitos alimentarios inadecuados en calidad y cantidad

- Consumo de tóxicos ( tabaco, alcohol y otras sustancias)

- Tatuajes

- Más de 2 parejas.

- Pelvis androide: pelvis antropoide, anomalías pelvianas pelvis platipeloide.

- Los problemas que se pueden presentar en los controles prenatales, se los puede agrupar en trimestres.

- Trastornos digestivos.

- Metrorragias.

- Abortos espontáneos.

- Embarazos extrauterinos.

\section{Análisis de resultados}

5.1 Casos de embarazos de alto riesgo identificados en las Unidades de Salud del Municipio de Langue, Valle tomando como base los años 2007 y 2009

En el municipio de Langue se está desarrollando un Proyecto de Descentralización de los Servicios de Salud; el cual es administrado por la Mancomunidad de Municipios Fronterizos "MAFRON". La implementación de este tipo de proyectos se hace con el objetivo de contribuir a la reducción de la morbilidad materna y la disminución de la morbilidad perinatal.

El municipio cuenta con 16 Unidades de Salud (US), ubicadas en las comunidades de las Olivas, Concepción de María, Potrerillos, San Marcos, Quebrada Grande, Agua Zarca, el Naranjo, Carrizal, San Francisco, Los Llanos, Llanitos Verdes, Papalón, Langue, Picacho y las Marías, de los cuales 5 US son Cesamos (Atendidos por doctor) y los restantes son Cesares (Atendidos por enfermeras).

La descentralización de los servicios de salud ayuda al descongestionamiento del sistema de la Secretaría de Salud; permitiendo con ello la contratación de médicos itinerantes, promotores y enfermeras auxiliares donde sean necesarios para lograr atender a las comunidades más alejadas de las Unidades de Salud ya establecidas como tal, puesto que el acceso a algunas unidades de salud es muy difícil y esto permite una mayor dificultad en la captación de partos institucionales de las mujeres embarazadas en las comunidades con poco o nulo acceso a los medios de transporte.

Para el año 2007 en el municipio de Langue se esperaba atender 726 partos de los cuales solamente el $27 \%$ fueron institucionales (atendidos en centros asistenciales) y el $26 \%$ fueron atendidos por parteras en sus comunidades presentando con ello un mayor grado de riesgo para los recién nacidos. Además de los 726 esperados no se registraron 388 partos que corresponde al 46\% (ver cuadro No.1).

Ese mismo año se detectaron 275 embarazos nuevos, de éstos 19 corresponden a adolecentes menores de 18 años. Una de las unidades de salud que atiende mayores casos de embarazos de adolecentes menores de 18 años de edad es la US del Cesamo de Langue el cual reportó el 53\% en adolescentes menores de 18 años.

Para el año 2009 en el municipio de Langue se esperaba atender 614 partos de los cuales solamente el 52\% fueron institucionales (atendidos en centros asistenciales), el $26 \%$ fue atendido por parteras en sus comunidades y no se registró, el $22 \%$ de partos que no fueron institucionales y tampoco fueron reportados por las parteras. 
Cuadro No. 1. Casos de embarazos de alto riesgo por Unidad de Salud del año 2007

\begin{tabular}{|c|c|c|c|c|c|c|c|c|}
\hline \multicolumn{9}{|c|}{ AÑO 2007} \\
\hline Unidad de Salud & $\begin{array}{l}\text { Partos Espe- } \\
\text { rados }\end{array}$ & $\begin{array}{c}\text { Partos } \\
\text { Institucionales }\end{array}$ & $\%$ & $\begin{array}{l}\text { Partos atendidos por } \\
\text { parteras }\end{array}$ & $\%$ & $\begin{array}{c}\text { Embarazos } \\
\text { Nuevos }\end{array}$ & $\begin{array}{c}\text { Embarazos en } \\
\text { menores de } 18 \\
\text { años }\end{array}$ & $\%$ \\
\hline Langue & 196 & 83 & 42 & 6 & 3 & 62 & 33 & 53 \\
\hline Las Olivas & 20 & 4 & 20 & 12 & 60 & 15 & 1 & 7 \\
\hline San Marcos & 44 & 5 & 11.3 & 20 & 45.5 & 27 & 2 & 7.4 \\
\hline Concepción de María & 70 & 9 & 13 & 50 & 71.5 & 39 & 2 & 5.2 \\
\hline Potrerillos & 36 & 11 & 315 & 11 & 30.5 & 8 & 0 & 0 \\
\hline El Naranjo & 38 & 18 & 47.5 & 10 & 26.3 & 32 & 2 & 6.3 \\
\hline Agua Zarca & 16 & 3 & 19 & 2 & 12.5 & 8 & 0 & 0 \\
\hline Los Llanos & 27 & 23 & 85 & 3 & 11 & 12 & 2 & 17 \\
\hline El Carrizal & 83 & 7 & 8.5 & 36 & 43.3 & 9 & 1 & 11 \\
\hline El Papalón & 44 & 7 & 16 & 3 & 7 & 6 & 0 & 0 \\
\hline Llanitos Verdes & 38 & 4 & 10.5 & 4 & 10.5 & 13 & 0 & 0 \\
\hline El Picacho & 26 & 6 & 23 & 8 & 31 & 10 & 1 & 10 \\
\hline Las Marías & 40 & 11 & 27.5 & 12 & 30 & 16 & 2 & 12.5 \\
\hline San Francisco & 48 & 9 & 19 & 11 & 23 & 18 & 3 & 17 \\
\hline Totales & 726 & 200 & $28 \%$ & 188 & $26 \%$ & 275 & 19 & $7 \%$ \\
\hline
\end{tabular}

Fuente: Secretaría de Salud/ Oficinas administrativas / Unidades de salud.

Ese mismo año 2009 se detectaron 513 nuevos embarazos de los cuales 50 de ellos fueron de adolecentes menores de 18 años; tal como se puede observar en el cuadro No.2. Las unidades de salud que atendieron el mayor número de embarazos de adolecentes menores de 18 años de edad fueron la US del Papalón y la US de San Marcos de Tamayo los cuales presentan un 27\% y 26\% de los embarazos respectivamente.

5.2 Análisis comparativos (años 2007 y 2009) de embarazos de alto riesgo detectados en las Unidades de Salud del municipio de Langue

Tal como se puede apreciar en el gráfico No.1 para el año 2009 aumentó el porcentaje de partos institucionales de un $28 \%$ a un $52 \%$ debido a una mayor captación de los embarazos que no estaban siendo reportados por las parteras ni por los centros de salud, podemos concluir que la concienciación directa e indirecta hacia las mujeres en estado de embarazo ha tenido mucha importancia.

Los grupos de edad que se consideran más afectados por los embarazos de alto riesgo son las mujeres menores de 20 años ya que estas aún dependen de su núcleo fami-
Gráfico No. 1. Municipio de Langue: embarazos de alto riesgo años 2007-2009

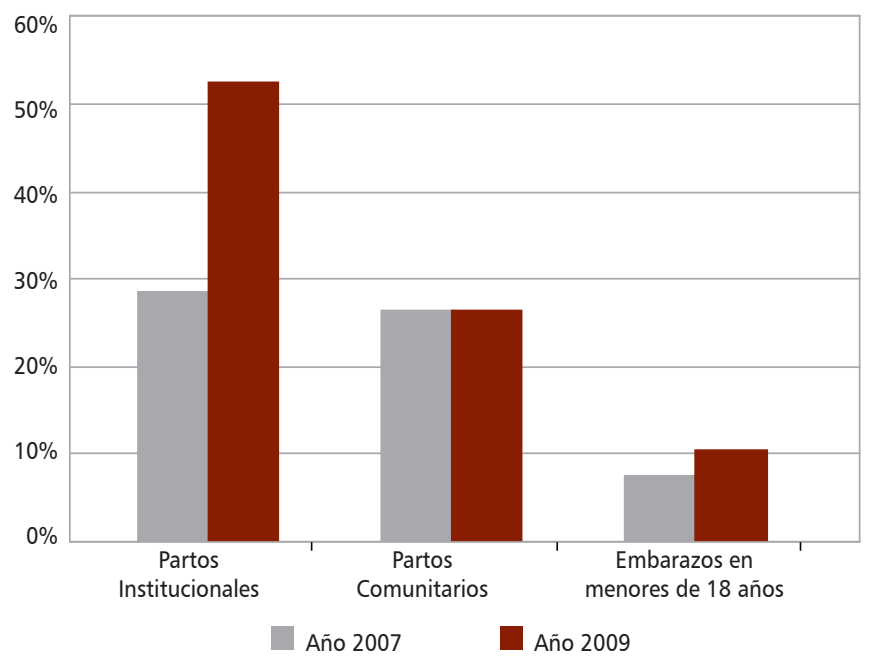

Fuente: Elaboración propia en base a datos de US, 2007-2009.

liar de origen por lo que no existe la madurez y conciencia emocional ni económica necesaria para implementar una adecuada prevención y cuidados oportunos en el proceso de embarazo; como también las mujeres mayores de 35 años considerando que existe el riesgo potencial de complicaciones obstétricas en dichas edades. 
Cuadro No. 2. Casos de embarazos de alto riesgo por Unidad de Salud del año 2009

\begin{tabular}{|c|c|c|c|c|c|c|c|c|}
\hline & & & & AÑO 2009 & & & & \\
\hline Unidad de Salud & $\begin{array}{c}\text { Partos } \\
\text { Esperados }\end{array}$ & $\begin{array}{c}\text { Partos } \\
\text { Institucionales }\end{array}$ & $\%$ & $\begin{array}{c}\text { Partos } \\
\text { atendidos por } \\
\text { parteras }\end{array}$ & $\%$ & $\begin{array}{c}\text { Embarazos } \\
\text { Nuevos }\end{array}$ & $\begin{array}{c}\text { Embarazos en } \\
\text { menores de } 18 \\
\text { años }\end{array}$ & $\%$ \\
\hline Langue & 176 & 112 & 64 & 4 & 2 & 133 & 12 & 9 \\
\hline Las Olivas & 20 & 5 & 25 & 6 & 30 & 11 & 0 & 0 \\
\hline San Marcos & 41 & 6 & 15 & 15 & 37 & 27 & 7 & 26 \\
\hline Concepción de María & 66 & 20 & 30 & 46 & 70 & 56 & 6 & 11 \\
\hline Potrerillos & 36 & 14 & 39 & 6 & 17 & 24 & 3 & 13 \\
\hline El Naranjo & 49 & 29 & 59 & 8 & 16 & 46 & 3 & 7 \\
\hline Agua Zarca & 12 & 7 & 58 & 0 & 0 & 13 & 0 & 0 \\
\hline Los Llanos & 32 & 22 & 69 & 2 & 6 & 31 & 4 & 13 \\
\hline El Carrizal & 49 & 18 & 37 & 19 & 39 & 41 & 3 & 7 \\
\hline El Papalón & 12 & 11 & 92 & 0 & 0 & 11 & 3 & 27 \\
\hline Llanitos Verdes & 19 & 6 & 32 & 7 & 37 & 19 & 0 & 0 \\
\hline El Picacho & 9 & 9 & 100 & 3 & 33 & 7 & 0 & 0 \\
\hline Las Marías & 35 & 37 & 106 & 4 & 11 & 39 & 3 & 8 \\
\hline San Francisco & 29 & 18 & 62 & 12 & 41 & 39 & 3 & 8 \\
\hline Quebrada Grande & 29 & 4 & 14 & 30 & 103 & 16 & 3 & 19 \\
\hline Totales & 614 & 318 & $52 \%$ & 162 & $26 \%$ & 513 & 50 & $10 \%$ \\
\hline
\end{tabular}

Fuente: Secretaría de Salud/ Oficinas administrativas / Unidades de salud

\subsection{Presencia institucional en las Unidades de Salud} apoyando directa e indirectamente a las mujeres embarazadas del municipio

En el municipio de Langue existen diversos programas que se encuentran apoyando el componente de salud con diversas actividades como la mejora de la calidad de sus servicios, su promoción, facilitación de alimentos, proyectos de agricultura, infraestructura, medio ambiente y capacitación comunitaria.
Las Unidades de Salud realizan diversas acciones para combatir los embarazos de alto riesgo en las adolecentes como ser: la captación de nuevos embarazos antes de las 12 semanas de gestación, el seguimiento continuo del embarazo, la consejería necesaria para los cuidados y tratamientos a seguir, dotación del medicamento necesario para el tratamiento de la embarazada, ofrecimiento de un espacio físico donde poder asistir el parto con los requerimientos mínimos que exige la Secretaría de Salud (ver Cuadro No.3).

Cuadro No. 3. Presencia institucional en las Unidades de Salud apoyando directa e indirectamente a las mujeres embarazadas del municipio

\begin{tabular}{|l|l|l|}
\multicolumn{1}{c|}{\begin{tabular}{c}
\multicolumn{1}{c|}{ Presencia } \\
Institucional
\end{tabular}} & \multicolumn{1}{|c|}{ Acciones } & \multicolumn{1}{c|}{ Unidades de Salud beneficiadas } \\
\hline Nueva Visión & $\begin{array}{l}\text { Apoya en la promoción de la salud, proyectos de agricultura, } \\
\text { crianza de animales, actividades de mejoras al medio ambiente, } \\
\text { letrinización y construcción de pilas. }\end{array}$ & $\begin{array}{l}\text { US de Langue, Potrerillos, El Naranjo, Agua Zarca, Los Llanos, Carrizal, } \\
\text { El Papalón, Llanitos Verdes, El Picacho, Las Marías, San Francisco }\end{array}$ \\
\hline $\begin{array}{l}\text { Programa Mundial } \\
\text { de Alimentos (PMA) }\end{array}$ & $\begin{array}{l}\text { Donación de alimentos mensual a las mujeres embarazadas, } \\
\text { lactantes y niños menores de } 5 \text { años de edad. }\end{array}$ & Las 15 Unidades de Salud del municipio de Langue, Valle. \\
\hline MAFRON & $\begin{array}{l}\text { Administración y ejecución del proyecto de los servicios de salud } \\
\text { descentralizados. }\end{array}$ & Las 15 Unidades de Salud del municipio de Langue, Valle. \\
\hline Mar y Mundo & $\begin{array}{l}\text { Atención integral a la mujer (Mediante los partos, los embarazos } \\
\text { y el puerperio). }\end{array}$ & US de Langue. \\
\hline
\end{tabular}

Fuente: Alcaldía Municipal/ Unidades de Salud del Municipio de Langue, Valle. 


\section{Conclusiones}

- En el municipio de Langue, departamento de Valle los casos de embarazos de alto riesgo para el año 2007 prevalecieron en la Unidad de Salud del Cesamo Langue con el $53 \%$ de los nuevos embarazos captados que se presenta en mujeres menores de 18 años de edad. Para el año 2009 las Unidades de Salud que prevalecieron son la US del Papalón con el 27\% y la US de San Marcos de Tamayo con un 26\% de los nuevos embarazos captados que se presenta en mujeres menores de 18 años de edad. Concluyendo con ello que en el 2009 la US de Langue trabajó fuertemente el tema de educación sexual brindando consejería y ofrecimiento de métodos de planificación familiar (uso adecuado de condones, DIU, etc.).

- La población femenina más afectada y vulnerable son las mujeres que se encuentran en el rango de edad menores de 20 años y mayores de 35 años (Grupos de edad económicamente activo). Reportándose para el año 2009; 50 embarazos en mujeres menores de 18 años.

- Las Unidades de Salud caracterizan los embarazos de alto riesgo como anticipatorios (Aplicar medidas preventivas a las mujeres embarazadas) e integrales (Considerar los aspectos biológicos, psicosociales y sociales de la mujer embarazada) por lo tanto, en este tipo de embarazos, siempre que se brinde el debido seguimiento y se controle el proceso por las partes involucradas podrá reducirse un mayor porcentaje del riesgo.

- El tipo de riesgo considerado por la Secretaría de Salud de Honduras más vulnerable para los embarazos de alto riesgo es el riesgo obstétrico y perinatal que contempla los antecedentes de patología médica obstétrica general, antecedentes de abusos sexuales, desnutrición, estaturas, niveles de educación, menarca a los 11 años o menos, actitudes, indiferencia a la importancia del embarazo, etc.

- De las Unidades de Salud estudiadas en el año 2007, dos presentan incidencia de partos comunitarios (fac- tor de riesgo) mayores al 50\% de los partos esperados; y en cuanto al año 2009, ninguna US presenta incidencia de riesgo. La incidencia del factor de riesgo antes mencionado se presenta en algunas circunstancias por el difícil acceso a las Unidades de Salud, la cultura y condición económica.

- En el año 2007 se registró el 28\% de partos institucionales, el 26\% de partos comunitarios y un 7\% de partos en adolecentes menores de 18 años que comparado con el número de embarazos esperados para ese año podemos concluir que un $48 \%$ de los partos no fueron registrados. En el año 2009 se registró el 52\% de partos institucionales, el 26\% de partos comunitarios y un 10\% de partos en adolecentes menores de 18 años que comparado con el número de embarazos esperados para ese año podemos concluir que un 22\% de los partos no fueron registrados. Por tanto es necesario resaltar que en relación a los dos años investigados se mejoró la captación y registro de los partos institucionales, se mantuvieron los porcentajes de partos comunitarios y se aumentó en promedio el 3\% de los embarazos en adolecentes menores de 18 años de edad.

\section{Recomendaciones}

- A todas las instituciones gubernamentales y no gubernamentales que trabajan apoyando a la Secretaría de Salud se les recomienda contemplar en sus objetivos planteados el componente de educación sexual; ya que una población educada en esta área permitirá un mayor desarrollo local.

- Que la Secretaría de Salud realice una revisión de los programas de educación sexual en cuanto a cobertura y calidad.

- Implementar un programa alternativo moderno de educación para padres que permita formar a sus hijos adecuadamente en el aspecto sexual.

- Por parte del gobierno local es necesario el apoyo a la organización comunitaria de mujeres y la conformación de la Oficina Municipal de la Mujer para que se brinde una atención integral a la mujer. 
- Implementar el sistema consultas externas y facilitación de medicamentos a las comunidades postergadas de las Unidades de Salud.

\section{Bibliografía}

- Secretaría de Salud (2007 y 2009). Oficinas administrativas de salud, Langue Valle.

- Maestría en Demografía Social- UNAH (2007). Diplomado en Objetivos de Desarrollo del Milenio con Énfasis en Análisis Sociodemográfico. Presentaciones en Power Point.

- Dirección Departamental de Salud de Valle (2011) Departamento de Epidemiología.
- Secretaría de Salud, (2010) 15 unidades de salud del municipio de Langue, Valle

- Naciones Unidas, segundo informe del país, Honduras 2007.

- Ruoti, A. M.(2000) Salud sexual y reproductiva del adolescente, Obstetricia y Perinatología, Cap. 8, 2aㅡ Edición EFACIM-EDUNA.

- Informe municipal de Langue Valle (2010). Sistema de Observatorios para el seguimiento de las metas del Milenio. Honduras.

- USAID, Fronteras de la Salud Reproductiva, (1998). Honduras Mejorar la Salud Reproductiva, Investigación Operativa. 
Anexo No.1. Acciones emprendidas por las Unidades de Salud del municipio para prevención de embarazos a temprana edad como factor de incidencia en los embarazos de alto riesgo

\begin{tabular}{|c|c|c|}
\hline Acciones emprendidas & Persona responsable & Observaciones \\
\hline $\begin{array}{l}\text { Control prenatal y puerperal de acuerdo a nor- } \\
\text { mas de la Secretaría de Salud. }\end{array}$ & Enfermera / Doctor & Se le brinda un seguimiento al crecimiento del niño, niña y a su madre \\
\hline Captación de embarazadas antes de 12 semanas & Promotor social & $\begin{array}{l}\text { El promotor social realiza un sondeo mensual en las comunidades para la } \\
\text { identificación de nuevos embarazos. }\end{array}$ \\
\hline Acción de espaciamiento & Enfermera & $\begin{array}{l}\text { La enfermera se encarga de aconsejar a la parturienta de la necesidad de } \\
\text { establecer espacios de tiempo entre un embarazo y otro. }\end{array}$ \\
\hline $\begin{array}{l}\text { Consejería y oferta de métodos de Planificación } \\
\text { Familiar (Depoprovera, Condones, DIU, ACO) }\end{array}$ & $\begin{array}{l}\text { Enfermera / Promotor } \\
\text { social }\end{array}$ & $\begin{array}{l}\text { La enfermera o promotor social brinda asesoría sobre la importancia del uso } \\
\text { de métodos de planificación a las mujeres embarazadas. }\end{array}$ \\
\hline $\begin{array}{l}\text { Manejo inicial de las complicaciones obstétricas } \\
\text { y neonatales }\end{array}$ & Enfermera & $\begin{array}{l}\text { Se realiza una revisión profunda del proceso de evolución de la parturienta. Y } \\
\text { de ser necesario se hace el respectivo traslado a un centro asistencial. (hospital) }\end{array}$ \\
\hline $\begin{array}{l}\text { Capacitación formal en atención integral a la } \\
\text { mujer }\end{array}$ & Enfermera & $\begin{array}{l}\text { Se le brinda capacitación a las parturientas sobre la importancia de mejor } \\
\text { uso y consumo de los alimentos. }\end{array}$ \\
\hline $\begin{array}{l}\text { Atención de parto institucional de acuerdo a } \\
\text { normas. }\end{array}$ & Doctor / Enfermera & $\begin{array}{l}\text { Atender los partos con los requerimientos mínimos que exige la Secretaría } \\
\text { de Salud. }\end{array}$ \\
\hline Suplementacion de acido fólico y prenatales & $\begin{array}{l}\text { Doctor / Enfermera/ } \\
\text { Secretaría de Salud }\end{array}$ & Brindar el medicamento necesario a las embarazadas y recién paridas. \\
\hline Control general de la morbilidad de la mujer. & Enfermera & $\begin{array}{l}\text { Conocer la situación general de la parturienta (Enfermedades que presenta } \\
\text { etc.) }\end{array}$ \\
\hline $\begin{array}{l}\text { Alojamiento conjunto Inmediato y mediato de } \\
\text { la parturienta }\end{array}$ & $\begin{array}{l}\text { Enfermera / Doctor / } \\
\quad \text { Promotor }\end{array}$ & $\begin{array}{l}\text { Brindar un espacio físico a la parturienta para poder ser atendida en un } \\
\text { centro asistencial. }\end{array}$ \\
\hline $\begin{array}{l}\text { Sensibilización sobre la importancia de la lactan- } \\
\text { cia materna exclusiva. }\end{array}$ & Enfermera & $\begin{array}{l}\text { Se le brinda asesoría sobre la importancia que tiene amamantar a los recién } \\
\text { nacidos. }\end{array}$ \\
\hline $\begin{array}{l}\text { Cuidados inmediatos y mediatos del recién } \\
\text { nacido }\end{array}$ & Doctor / Enfermera & Se trabaja más que todo con adolecentes (Mujeres primerizas). \\
\hline $\begin{array}{l}\text { Toma de citologías y seguimiento casos anor- } \\
\text { males }\end{array}$ & $\begin{array}{l}\text { Doctor / Licenciadas de } \\
\text { enfermería }\end{array}$ & $\begin{array}{l}\text { Se realizan citologías generales a las mujeres que padecen de malestares } \\
\text { continuos (Infecciones vaginales). }\end{array}$ \\
\hline $\begin{array}{l}\text { Atención integral y primordial al niño menor } \\
\text { de } 2 \text { años }\end{array}$ & Enfermera & Administración y seguimiento de las vacunas. \\
\hline $\begin{array}{l}\text { Educación sexual y reproductiva (Generó, va- } \\
\text { lores, autoestima, prevención de ITS / VIH / SIDA) }\end{array}$ & Promotor social & $\begin{array}{l}\text { Se educa a las comunidades sobre la importancia de los valores morales en } \\
\text { las familias, la autoestima de las mujeres y la igualdad de tratos tanto para } \\
\text { las mujeres como también para los hombres. }\end{array}$ \\
\hline Atención y prioridad de emergencias & Doctor / Enfermera & Facilitación de medios para la movilización de emergencias. \\
\hline Ferias de la salud & Personal de salud & $\begin{array}{l}\text { Brindar consultas externas y medicamentos en fechas determinadas y con } \\
\text { mucha concurrencia de personas. }\end{array}$ \\
\hline Educación en salud en las escuelas y colegios & Promotor social & $\begin{array}{l}\text { Asistir a los centros educativos a brindar charlas sobre la importancia y } \\
\text { cuidados a la salud. }\end{array}$ \\
\hline $\begin{array}{l}\text { Organización y seguimiento a círculos de em- } \\
\text { barazadas }\end{array}$ & $\begin{array}{l}\text { Enfermera / Promotor } \\
\text { social }\end{array}$ & Organización por grupos de riesgos \\
\hline $\begin{array}{l}\text { Notificación e Investigación de la mortalidad } \\
\text { materna e infantil }\end{array}$ & Promotor Social & Informar a la Secretaría de Salud \\
\hline $\begin{array}{l}\text { Notificación y seguimiento de casos de embara- } \\
\text { zos con VIH/Sida }\end{array}$ & Promotor social & $\begin{array}{l}\text { Informar a los superiores sobre los casos de VIH/SIDA y facilitar el medicamento } \\
\text { necesario a las parturientas. }\end{array}$ \\
\hline $\begin{array}{l}\text { Sistema de referencia y respuesta entre centros } \\
\text { asistenciales. }\end{array}$ & $\begin{array}{l}\text { Secretaría de Salud / } \\
\text { Regional de Salud }\end{array}$ & Dar seguimiento a los casos remetidos a otros centros asistenciales. \\
\hline $\begin{array}{l}\text { Elaboración y actualización de mapas de riesgos } \\
\text { basados en los censos, encuestas y otros medios } \\
\text { para control y seguimiento de los grupos de } \\
\text { riesgo (embarazos, hipertensión, etc.) }\end{array}$ & Promotor Social & Identificación gráfica de los grupos de riesgo presentes en la comunidad. \\
\hline Visitas domiciliar a familias de riesgo & $\begin{array}{l}\text { Promotor social/ } \\
\text { Enfermera }\end{array}$ & Realizar visitas domiciliarias a las familias con grupos de riesgos. \\
\hline $\begin{array}{l}\text { Entrega de paquete básico a comunidades de } \\
\text { difícil acceso. }\end{array}$ & Personal de Salud & $\begin{array}{l}\text { Brindar consultas externas y medicamentos a las comunidades postergadas } \\
\text { de las unidades de salud. }\end{array}$ \\
\hline
\end{tabular}

Fuente: Unidades de Salud del Municipio de Langue, Valle 